\title{
Three-loop gauge beta function in non-simple gauge groups
}

\author{
Luminita Mihaila*t \\ TTP Karlsruhe Institute for Technology \\ E-mail: Luminita-Nicoletta.Mihaila@kit.edu
}

In this talk, I report on the recent calculation of the three-loop gauge beta functions for a nonsimple gauge group. The result can be compactly expressed in terms of quadratic Casimir invariants and rational numbers. Comparisons with the existing calculations for the Standard Model (SM) and its Minimal Supersymmetric Extension (MSSM) are reviewed.

11th International Symposium on Radiative Corrections (Applications of Quantum Field Theory to Phenomenology) (RADCOR 2013),

22-27 September 2013

Lumley Castle Hotel, Durham, UK

\footnotetext{
* Speaker.

${ }^{\dagger}$ The author is grateful to Luca Di Luzio, Jens Salomon and Maathias Steinhauser for successful collaboration.
} 


\section{Introduction}

Beta functions are fundamental quantities in any quantum field theory. They provide us with information about the behavior of the theory at very high or very low energies. Therefore, they reveal the possible phase transitions that the system can undergo and set limits on the validity of the perturbative methods, that we heavily relay on. The computations of beta functions for nonabelian Yang-Mills theories has a long history [1,2], being among the first radiative corrections ever achieved in such frameworks. It has lead to the discovery of the asymptotic freedom and contributed decisively to the development of Quantum Chromodynamics (QCD) as the theory of strong interaction.

Currently, the beta functions of QCD are known to the fourth loop-order [3, 4] and even the first contributions at the fifth loop became recently available [5]. They are essential ingredients for the extraction of the strong coupling constant at the $Z$-boson mass from the experimental data. They also are required for the prediction of the hadronic Higgs production cross section and decay rate at an accuracy level compatible with the experimental.

For the electroweak sector of the SM, the situation is slightly less evolved. The three-loop computation of the beta functions for all SM couplings (gauge [6, 7], Yukawa [8, 9] and Higgs selfcoupling $[10,11])$ was completed only during the last two years. This delay can be explained by the huge number of diagrams that have to be calculated, by the more complicated infrared behavior of the SM as compared to QCD and by the issue of spontaneous gauge symmetry breaking in the SM (i.e. transition from the unbroken to the broken phase). Let us mention at this point, that these recent calculations enabled the reduction of the theoretical uncertainty for the prediction of the electroweak gauge couplings at a level compatible with the current experimental accuracy. In addition, they are essential ingredients for the high precision analysis of the electroweak vacuum stability, especially after the discovery of the Higgs boson and the measurement of its mass at per mill level.

For supersymmetric extension of the standard model, the three-loop gauge beta functions are available already since long time [12], however the underlying computation is not based on component field methods and resides heavily on the supersymmetric nature of the theory. A component field confirmation of these results is presently available only for the Supersymmetric Quantum Chromodynamics (SQCD) [13].

For non-supersymmetric extensions of the SM, there is the computation of the three-loop gauge beta functions for a simple gauge group [14] with the obvious application to unified gauge theories (GUT). However, any GUT beyond the minimal SU(5) model predicts a tower of heavy particles between the electroweak- and the Planck-scale. For widely separated mass spectra, one has to introduce effective theories valid at the various mass scales of the theory and use the Renormalization Group Equations (RGEs) to relate predictions for physical observables at different scales. Necessarily, the RGEs require the knowledge of the gauge beta functions also for non-simple gauge groups. The two-loop contributions are known since long [15] and have a very compact expression in terms of Casimir invariants. In this talk, I report on the calculation of the three-loop contributions the gauge beta functions for non-simple gauge groups, taking into account gauge, Yukawaand scalar interactions [16]. 


\section{Calculation}

Let us consider a quantum field theory based on a non-simple gauge group defined as a direct product of $n$ simple groups $G=G_{1} \otimes G_{2} \cdots \otimes G_{n}$. For generality, we consider each simple group as non-abelian. The case of abelian groups can be easily derived from the general results by changing the Casimir invariants accordingly. The particle content is made of $n$-types of gauge bosons, Dirac and Majorana fermios, that for generality are chosen to be charged w.r.t. all the simple gauge groups ${ }^{1}$, and two-types of scalars, that again for completeness are charged under all the simple gauge groups. We choose two-types of scalars for simplicity and easiness of bookkeeping in latter applications: i) To distinguish the scalars that develop a vacuum expectation value, for future analysis related with spontaneous symmetry breaking; ii) To introduce scalars that may live in other than $D=4-2 \varepsilon$ dimensions for latter use in supersymmetric theories. For example, in case of the SM regulated in Dimensional Reduction, one scalar multiplet will describe the SM Higgsdoublet and the other multiplet represents the $\varepsilon$-scalars.

Since we want to compute the gauge beta functions at three-loops, the maximal number of different gauge bosons that can appear in one diagram is, of course, equal to three. Thus, in practical calculations it is enough to work with a direct product of three different simple gauge groups.

For phenomenological applications, it is important to take into considerations also chiral theories. Because of the issue of $\gamma_{5}$ in higher order computations, we work for the very beginning with chiral fermions, defining as usual the left- and right-handed components $F_{L}=P_{L} F$ and $F_{R}=P_{R} F$, with $P_{L / R}=\frac{1 \mp \gamma_{5}}{2}$. This setup translates into modified Feynman rules for vertices involving fermions and a larger number of diagrams as compared to the minimal possible choice for non-chiral theories. Nevertheless, we can use this artificial separation of the fermionic degrees of freedom as an internal check for the treatment of $\gamma_{5}$ at three-loop order.

For completeness, let us introduce at this point the Lagrangian that describe this theory:

$$
\mathscr{L}=\mathscr{L}_{\mathrm{YM}}+\mathscr{L}_{\text {fix }}+\mathscr{L}_{\mathrm{FP}}+\mathscr{L}_{\mathrm{F}}+\mathscr{L}_{\mathrm{S}}+\mathscr{L}_{\text {Yuk }}
$$

where the first three terms describe the pure gauge Lagrangian, $\mathscr{L}_{\mathrm{F}}$ stands for the genuine fermion contribution, $\mathscr{L}_{\mathrm{S}}$ denotes the scalar Lagrangian, and the last term takes into account Yukawa interactions defined as the interaction between a scalar and two fermions. Explicitly,

$$
\mathscr{L}_{\mathrm{YM}}=-\frac{1}{4} \sum_{i=1}^{n} F_{\mu \nu}^{a, i} F^{\mu v, a, i}, \quad F_{\mu \nu}^{a, i}=\partial_{\mu} W_{v}^{a, i}-\partial_{\nu} W_{\mu}^{a, i}+g_{i} f^{a b c, i} W_{\mu}^{b, i} W_{v}^{c, i}
$$

where $W \mu^{a, i}$ is the gauge field, $f^{a b c, i}$ denotes the structure constant and $g_{i}$ gives the coupling constant for the gauge group $G_{i}$. For the gauge fixing term $\mathscr{L}_{\text {fix }}$ and the associated Fadeev-Popov ghost contributions $\mathscr{L}_{\text {FP }}$ we chose the Lorenz gauge.

The fermionic contribution reads

$$
\mathscr{L}_{\mathrm{F}}=i \sum_{f=F_{L}, F_{R}}^{N_{F}} \bar{\psi}_{f} \not \supset \psi_{f}, \quad D_{\mu, f}=\partial_{\mu}-i \sum_{i=1}^{n} g_{i} R_{f}^{a, i} W_{\mu}^{a, i},
$$

\footnotetext{
${ }^{1}$ The case of non-interaction with some of the $n$-types of gauge bosons is obviously obtained by nullifying their charge w.r.t. the corresponding groups.
} 
where $\Psi_{f}$ denotes the fermion fields, $F_{f}^{a, i}$ is the generator of the group $G_{i}$ in the fermionic representation $f$. The sum over $f$ runs over all fermion species and the sum over $i$ encounters all the gauge groups.

The scalar Lagrangian is given by

$$
\mathscr{L}_{\mathrm{S}}=\sum_{s}\left(D_{\mu} \Phi_{s}\right)^{\dagger}\left(D^{\mu} \Phi_{s}\right)+V(\Phi)
$$

with the covariant derivative defined now for the group generators in the scalar representation $S_{s}^{a, i}$. For the calculation of the gauge beta functions, only the quartic terms from the potential are of interest, as can be easily understood from dimension counting. For the model under discussion, they are described by the following potential

$$
V_{4 \mathrm{sc}}=\lambda_{1}\left(\Phi_{1}^{\dagger} \Phi_{1}\right)^{2}+\lambda_{2}\left(\Phi_{2}^{\dagger} \Phi_{2}\right)^{2}+\lambda_{12}\left(\Phi_{1}^{\dagger} \Phi_{1}\right)\left(\Phi_{2}^{\dagger} \Phi_{2}\right),
$$

where $\lambda_{k}, k=1,2,12$ denotes the quartic self and mixed scalar couplings.

Furthermore, all the group theoretical factors we encountered in the three-loop order calculation can be expressed in terms of quadratic Casimir invariants of the relevant representations of the gauge group. For a field transforming under the representation $R$ of the gauge group $G$, where the generators $R^{A}$ satisfy

$$
\left[R^{A}, R^{B}\right]=i f^{A B C} R^{C},
$$

the Casimir invariants are defined as follows

$$
\begin{aligned}
\operatorname{Tr}\left(R^{A} R^{B}\right) & =\delta^{A B} T(R), & & R_{a c}^{A} R_{c b}^{A}=\delta_{a b} C(R), \\
f^{A C D} f^{B C D} & =\delta^{A B} C(G), & & \delta^{A A}=d(G) .
\end{aligned}
$$

Here $d(G)$ denotes the dimension of the group. Then the following relation holds as well

$$
C(R) d(R)=T(R) N(G)
$$

where $d(R)=\delta_{a a}$ is the dimension of representation $R$. In our final results we use the following notation $d\left(R_{i}\right)$, to specify the multiplicity of the representation $R$ w.r.t. the gauge group $G_{i}$, with $R=F, S$. For later convenience, we define also the multiplicity (dimension) of a representation w.r.t a subset of the original direct product of simple groups as :

$$
D\left(R_{i}\right)=\prod_{\substack{j \neq i \\ j=1}}^{n} d\left(R_{j}\right), \quad D\left(R_{i j}\right)=\prod_{\substack{k \neq i, j \\ k=1}}^{n} d\left(R_{k}\right), \quad \text { and } \quad D\left(R_{i j k}\right)=\prod_{\substack{l \neq i, j, k \\ l=1}}^{n} d\left(R_{l}\right)
$$

with $R=F, S$.

The coupling constants are expressed in terms of the corresponding gauge couplings through the relations: $\alpha_{i}=g_{i}^{2} /(4 \pi)$ with $i=1,2, \ldots, n$. The beta functions are defined as

$$
\begin{aligned}
& \mu^{2} \frac{d}{d \mu^{2}} \frac{\alpha_{i}}{\pi}=\beta_{i}\left(\left\{\alpha_{j}\right\}, \varepsilon\right)=-\varepsilon \frac{\alpha_{i}}{\pi} \\
& -\left(\frac{\alpha_{i}}{\pi}\right)^{2}\left[a_{i}+\sum_{j} \frac{\alpha_{i}}{\pi} b_{i j}+\sum_{j, k} \frac{\alpha_{j}}{\pi} \frac{\alpha_{k}}{\pi} c_{i j k}+\ldots\right],
\end{aligned}
$$


with $i=1,2, \ldots, n$. The expression after the second equality sign gives the perturbative expansion.

In practice, the functions $\beta_{i}$ are obtained from the renormalization constants of the corresponding couplings that are defined as $\alpha_{i}^{\text {bare }}=\mu^{2 \varepsilon} Z_{\alpha_{i}} \alpha_{i}$. Exploiting the fact that the bare couplings are $\mu$-independent and taking into account that $Z_{\alpha_{i}}$ may depend on all the other couplings leads to the following formula

$$
\beta_{i}=-\left[\varepsilon \frac{\alpha_{i}}{\pi}+\frac{\alpha_{i}}{Z_{\alpha_{i}}} \sum_{j, j \neq i}^{7} \frac{\partial Z_{\alpha_{i}}}{\partial \alpha_{j}} \beta_{j}\right]\left(1+\frac{\alpha_{i}}{Z_{\alpha_{i}}} \frac{\partial Z_{\alpha_{i}}}{\partial \alpha_{i}}\right)^{-1},
$$

From Eq. (2.11) it is clear that the renormalization constants $Z_{\alpha_{i}}(i=1,2,3)$ have to be computed up to three-loop order. In principle each vertex containing the gauge coupling $\alpha_{i}$ at tree level can be used in order to obtain $Z_{\alpha_{i}}$ via

$$
Z_{\alpha_{i}}=\frac{\left(Z_{\mathrm{vrtx}}\right)^{2}}{\prod_{k} Z_{k, \mathrm{wf}}}
$$

where $Z_{\mathrm{vrtx}}$ stands for the renormalization constant of the vertex and $Z_{k, \mathrm{wf}}$ for the wave function renormalization constant; $k$ runs over all external particles.

We have computed $Z_{\alpha_{i}}$ using the ghost-gauge boson vertices as they are the most economical ones w.r.t. number of diagrams. For abelian gauges, the renormalization constant for ghost-gauge boson vertex vanishes through three loops, that is a valuable consistency check of the setup. For comparison with beta functions in super-symmetric theories, we also computed the Yukawa coupling renormalization at three-loop order.

For the present calculation, we use a well-tested chain of programs: QGRAF [17] generates all contributing Feynman diagrams. The output is passed via q2e [18, 19], which transforms Feynman diagrams into Feynman amplitudes, to exp $[18,19]$ that generates FORM[20] code. The latter is processed by MINCER [21] that computes analytically massless propagator diagrams up to three loops and outputs the $\varepsilon$ expansion of the result.

\section{Results}

The three-loop gauge beta function reads

$$
\begin{aligned}
\beta_{\alpha_{i}} & =-\varepsilon \frac{\alpha_{i}}{\pi}+\left(\frac{\alpha_{i}}{\pi}\right)^{2} \frac{1}{4}\left[-\frac{11}{3} C\left(G_{i}\right)+\sum_{F} \frac{2}{3} T\left(F_{i}\right) D\left(F_{i}\right)+\sum_{S} \frac{1}{3} T\left(S_{i}\right) D\left(S_{i}\right)\right] \\
& +\left(\frac{\alpha_{i}}{\pi}\right)^{2} \frac{1}{16}\left\{\frac { \alpha _ { i } } { \pi } \left[-\frac{34}{3} C\left(G_{i}\right)^{2}+\sum_{F}\left[\frac{10}{3} C\left(G_{i}\right)+2 C\left(F_{i}\right)\right] T\left(F_{i}\right) D\left(F_{i}\right)\right.\right. \\
& \left.+\sum_{S}\left[\frac{2}{3} C\left(G_{i}\right)+4 C\left(S_{i}\right)\right] T\left(S_{i}\right) D\left(S_{i}\right)\right]+\sum_{j \neq i} \frac{\alpha_{j}}{\pi}\left[\sum_{F} 2 C\left(F_{j}\right) d\left(F_{j}\right) T\left(F_{i}\right) D\left(F_{i j}\right)\right. \\
& \left.\left.+\sum_{S} 4 C\left(S_{j}\right) d\left(S_{j}\right) T\left(S_{i}\right) D\left(S_{i j}\right)\right]+\sum_{F}\left[-T\left(F_{i}\right) \frac{1}{\pi} \operatorname{Tr}\left(\mathrm{Y}_{\mathrm{F}_{\mathrm{R}}} \mathrm{Y}_{\mathrm{F}_{\mathrm{R}}^{\dagger}}^{\dagger}\right)\right]\right\} \\
& +\left(\frac{\alpha_{i}}{\pi}\right)^{3} \frac{1}{64}\left\{\frac { \alpha _ { i } } { \pi } \left[-\frac{2857}{54} C\left(G_{i}\right)^{3}+\sum_{F}\left[\frac{1415}{54} C\left(G_{i}\right)^{2}+\frac{205}{18} C\left(G_{i}\right) C\left(F_{i}\right)-C\left(R_{i}\right)^{2}\right] T\left(F_{i}\right) D\left(F_{i}\right)\right.\right. \\
& +\sum_{S}\left[\frac{545}{108} C\left(G_{i}\right)^{2}+\frac{1129}{36} C\left(G_{i}\right) C\left(S_{i}\right)+\frac{29}{2} C\left(S_{i}\right)^{2}\right] T\left(S_{i}\right) D\left(S_{i}\right)
\end{aligned}
$$




$$
\begin{aligned}
& -\sum_{F} \sum_{S}\left[\frac{29}{27} C\left(G_{i}\right)+\frac{23}{18} C\left(F_{i}\right)+\frac{25}{9} C\left(S_{i}\right)\right] T\left(R_{i}\right) T\left(S_{i}\right) D\left(F_{i}\right) D\left(S_{i}\right) \\
& -\sum_{F_{m}, F_{n}}\left[\frac{79}{54} C\left(G_{i}\right)+\frac{11}{9} C\left(F_{m, i}\right)\right] T\left(F_{m, i}\right) T\left(F_{n, i}\right) D\left(F_{m, i}\right) D\left(F_{n, i}\right) \\
& \left.+\sum_{S_{m}, S_{n}}\left[\frac{1}{27} C\left(G_{i}\right)-\frac{49}{18} C\left(S_{m, i}\right)\right] T\left(S_{m, i}\right) T\left(S_{n, i}\right) D\left(S_{m, i}\right) D\left(S_{n, i}\right)\right] \\
& +\sum_{j \neq i} \frac{\alpha_{j}}{\pi}\left[\sum_{F} 2\left[2 C\left(G_{i}\right)-C\left(F_{i}\right)\right] T\left(R_{i}\right) C\left(F_{j}\right) D\left(F_{i j}\right)+\sum_{S}\left[\frac{25}{2} C\left(G_{i}\right)+29 C\left(S_{i}\right)\right] T\left(S_{i}\right) C\left(S_{j}\right) D\left(S_{i j}\right)\right] \\
& +\left(\frac{\alpha_{i}}{\pi}\right)^{2} \frac{1}{64}\left\{\sum_{j \neq k} \frac{\alpha_{j}}{\pi} \frac{\alpha_{k}}{\pi}\left[-\sum_{F} C\left(F_{j}\right) C\left(F_{k}\right) T\left(F_{i}\right) D\left(F_{i j k}\right)-\sum_{S} 2 C\left(S_{j}\right) C\left(S_{k}\right) T\left(S_{i}\right) D\left(S_{i j k}\right)\right]\right. \\
& +\sum_{j}\left(\frac{\alpha_{j}}{\pi}\right)^{2}\left[\sum_{F}\left[\frac{133}{18} C\left(G_{j}\right)-C\left(F_{j}\right)\right] C\left(F_{j}\right) T\left(F_{i}\right) D\left(F_{i j}\right)\right. \\
& +\sum_{S}\left[\frac{679}{36} C\left(G_{j}\right)+\frac{29}{2} C\left(S_{j}\right)\right] C\left(S_{j}\right) T\left(S_{i}\right) D\left(S_{i j}\right) \\
& -\sum_{F_{m}, F_{n}} \frac{11}{9} C\left(F_{m, j}\right) T\left(F_{n, j}\right) T\left(F_{m, i}\right) D\left(F_{m, i j}\right) D\left(F_{n, j}\right)-\sum_{S_{m}, S_{n}} \frac{49}{18} C\left(S_{m, j}\right) T\left(S_{n, j}\right) T\left(S_{m, i}\right) D\left(S_{m, i j}\right) d\left(S_{n, j}\right) \\
& \left.-\sum_{S} \sum_{F}\left[\frac{25}{9} C\left(S_{j}\right) T\left(S_{i}\right) T\left(F_{j}\right)+\frac{23}{18} C\left(F_{j}\right) T\left(F_{i}\right) T\left(S_{j}\right)\right] d\left(F_{i j}\right) d\left(S_{i j}\right)\right]+\beta_{2}^{\mathrm{Yuk}}+\beta_{2}^{4 \mathrm{sc}},
\end{aligned}
$$

where the last two terms the contributions from Yukawa and scalar quartic interactions denote. They do not factorise as is the case for the pure gauge contributions and require a dedicated analysis $[14,16]$. The can be actually easily derived from the results of Ref. [14], via obvious replacements. We compared the above results with explicit calculations for the SM [6, 7], SU(5)+24 [22], and SQC $[12,13]$ and found complete agreement.

Let us stress at the point the absence in the beta functions of transcendental numbers and of higher Casimir invariants, although they are present at the diagram level. Another interesting point is that the semi-naive treatment of $\gamma_{5}$ as proposed in Ref. [23] is sufficient also for this calculation.

\section{References}

[1] D. J. Gross and F. Wilczek, Ultraviolet Behavior of Nonabelian Gauge Theories, Phys. Rev. Lett. 30 (1973) 1343.

[2] H. D. Politzer, Reliable Perturbative Results for Strong Interactions?, Phys. Rev. Lett. 30 (1973) 1346.

[3] T. van Ritbergen, J. A. M. Vermaseren and S. A. Larin, The Four loop beta function in quantum chromodynamics," Phys. Lett. B 400 (1997) 379 [hep-ph/ 9701390 ].

[4] M. Czakon, The Four-loop QCD beta-function and anomalous dimensions, Nucl. Phys. B 710 (2005) 485 [hep-ph/ 0411261$].$

[5] K. Chetyrkin, contributions to thesse proceedings.

[6] L. N. Mihaila, J. Salomon and M. Steinhauser, Gauge Coupling Beta Functions in the Standard Model to Three Loops, Phys. Rev. Lett. 108 (2012) 151602 [arXiv:1201.5868 [hep-ph] ]. 
[7] A. V. Bednyakov, A. F. Pikelner and V. N. Velizhanin, Anomalous dimensions of gauge fields and gauge coupling beta-functions in the Standard Model at three loops, JHEP 1301 (2013) 017 [arXiv:1210.6873 [hep-ph]].

[8] K. G. Chetyrkin and M. F. Zoller, Three-loop beta-functions for top-Yukawa and the Higgs self-interaction in the Standard Model, JHEP 1206 (2012) 033 [arXiv: 1205.2892 [hep-ph] ].

[9] A. V. Bednyakov, A. F. Pikelner and V. N. Velizhanin, Yukawa coupling beta-functions in the Standard Model at three loops, Phys. Lett. B 722 (2013) 336 [arXiv: 1212 . 6829].

[10] K. G. Chetyrkin and M. F. Zoller, $\beta$-function for the Higgs self-interaction in the Standard Model at three-loop level, JHEP 1304 (2013) 091 [Erratum-ibid. 1309 (2013) 155] [arXiv: 1303.2890 [hep-ph] ].

[11] A. V. Bednyakov, A. F. Pikelner and V. N. Velizhanin, Higgs self-coupling beta-function in the Standard Model at three loops, Nucl. Phys. B 875 (2013) 552 [arXiv: 1303 . 4364].

[12] I. Jack, D. R. T. Jones and C. G. North, N=1 supersymmetry and the three loop gauge Beta function, Phys. Lett. B 386 (1996) 138 [hep-ph/9606323].

[13] R. V. Harlander, L. Mihaila and M. Steinhauser, The SUSY-QCD beta function to three loops, Eur. Phys. J. C 63 (2009) 383 [arXiv:0905.4807 [hep-ph] ].

[14] A. G. M. Pickering, J. A. Gracey and D. R. T. Jones, Three loop gauge beta function for the most general single gauge coupling theory, Phys. Lett. B 510 (2001) 347 [hep-ph / 0104247 ].

[15] D. R. T. Jones, The Two Loop beta Function for a G(1) x G(2) Gauge Theory, Phys. Rev. D 25 (1982) 581.

[16] L. Mihaila, Three-loop gauge beta function in non-simple gauge groups, in progress.

[17] , P. Nogueira, Automatic Feynman graph generation, J.Comput.Phys. 105 (1993) 279-289.

[18] R. Harlander, and T. Seidensticker, and M. Steinhauser", Complete corrections of Order alpha alpha-s to the decay of the Z boson into bottom quarks, Phys.Lett. B 426 (1998), 125-132, [hep-ph/9712228].

[19] T. Seidensticker, Automatic application of successive asymptotic expansions of Feynman diagrams", [hep-ph/9905298], TTP-99-22.

[20] J.A.M. Vermaseren, New features of FORM, (2000), [math-ph/0010025].

[21] S.A. Larin, F.V. Tkachov and J.A.M. Vermaseren, The FORM version of MINCER, (1991), [NIKHEF-H-91-18].

[22] L. Di Luzio and L. Mihaila, Unification scale vs. electroweak-triplet mass in the $S U(5)+24_{F}$ model at three loops, Phys. Rev. D 87 (2013) 115025 [arXiv:1305.2850 [hep-ph] ].

[23] L. N. Mihaila, J. Salomon and M. Steinhauser, Renormalization constants and beta functions for the gauge couplings of the Standard Model to three-loop order, Phys. Rev. D 86 (2012) 096008 [arXiv:1208.3357 [hep-ph]]. 